\title{
ADECUACIÓN CULTURAL Y CAPACITACIÓN ACERCA DEL PROCESO DE CONSENTIMIENTO INFORMADO EN PROYECTO SOBRE NUTRICIÓN ESCOLAR EN UN PUEBLO INDÍGENA AYMARA DE PERÚ
} CULTURAL ADEQUACY AND TRAINING WITH RESPECT TO THE INFORMED CONSENT PROCESS IN A SCHOOL NUTRITION PROJECT FOR AN AYMARA INDIGENOUS COMMUNITY IN PERU ADEQUAÇÃO CULTURAL E CAPACITAÇÃO ACERCA DO PROCESSO DE CONSENTIMENTO INFORMADO EM PROJETO SOBRE NUTRIÇÃO ESCOLAR NUM POVO INDÍGENA DO PERU

Agueda Muñoz del Carpio Toia ${ }^{1}$

Dueñas Ancco André2 Sánchez Rodríguez Kristell ${ }^{3}$

Begazo Muñoz Lucia ${ }^{4}$

\begin{abstract}
Resumen
Antecedentes: es importante que las investigaciones respeten normas éticas y la toma del consentimiento informado (CI) con la debida adecuación cultural y verificando su comprensión en comunidades indígenas de las regiones Andina y Amazónica, incluidas hoy en investigaciones sociales y médicas. Objetivo: determinar el grado de comprensión del CI en una comunidad aymara del Perú con un proyecto de nutrición escolar. Metodología: se realizó un estudio de campo cuasi experimental, prospectivo, de intervención educativa en Puno, en 2015. Se explicó a los líderes aymaras los objetivos y procedimientos del estudio de nutrición escolar y CI. Se confeccionó un cuestionario de conocimientos y prácticas de loncheras saludables para niños aymaras; un programa educa-
\end{abstract}

DOI: 10.5294/PEBI.2017.21.1.6

\section{PARA CITAR ESTE ARTÍCULO / TO REFERENCE THIS ARTICLE / PARA CITAR ESTE ARTIGO}

Muñoz del Carpio Toia A., Dueñas Ancco A., Sánchez Rodríguez K., Begazo Muñoz L. Adecuación cultural y capacitación acerca del proceso de consentimiento informado en proyecto sobre nutrición escolar en un pueblo indígena aymara de Perú. pers.bioét. 2017;21(1): 78-91. DOI: 10.5294/pebi.2017.21.1.6

1 orcid.org/ 0000-0003-2723-3544. Universidad Católica de Santa María, Perú. amunozde@ucsm.edu.pe

2 orcid.org/0000-0002-8052-6840. Universidad Católica de Santa María, Perú.dreyniko@gmail.com

3 orcid.org/0000-0003-2236-0536. Universidad Católica de Santa María,Perú.kmsr43@gmail.com

4 orcid.org/0000-0002-0262-0196. Universidad Católica de Santa María, Perú.luciatoia@gmail.com
FECHA DE RECEPCIÓN: 2016-10-09 FECHA DE ENVÍO A PARES: 2016-10-12 FECHA DE APROBACIÓN POR PARES: 2017-01-05 FECHA DE ACEPTACIÓN: 2017-01-09 
tivo de nutrición escolar y dos formatos de CI (simple y con adecuación cultural). La documentación fue evaluada por un comité de ética de la investigación. Resultados: 70 pobladores aymaras participaron del proyecto de investigación. El grado de comprensión del CI se modificó con significancia estadística luego de la intervención educativa y la adecuación cultural.

Palabras Clave: comprensión; consentimiento informado; pueblos indígenas; aymaras; investigación (Fuente: DeCS, Bireme).

\section{ABSTRACT}

Background: Research must respect ethical norms and informed consent (IC), with appropriate cultural adaptation and verification of its understanding in indigenous communities of the Andean and Amazonian regions, including social and medical research. Objective: Determine to what extent IC is understood in an Aymara community in Peru, specifically as concerns a school nutrition project. Methodology: A prospective, quasi-experimental field study of an educational intervention was conducted in Puno during 2015. The objectives and procedures of the school nutrition project in question were explained to the local Aymara leaders, and a questionnaire was prepared on knowledge and practices regarding healthy lunch boxes for Aymara children. An educational nutrition program and two IC formats (simple and culturally appropriate) were drawn up. The documentation was evaluated by a research ethics committee. Results: Seventy (70) Aymara villagers participated in the research project. The extent to which they understood the IC was modified to a statistically significant degree subsequent to the educational intervention and adaption for cultural appropriateness.

KeYwORDs: Understanding; informed consent; Indigenous peoples; the Aymara people; research (Source: DeCS, Bireme).

RESUMO

Antecedentes: é importante que as pesquisas respeitem normas éticas e a tomada do consentimento informado (CI) com a devida adequação cultural e verificando sua compreensão em comunidades indígenas das regiões andina e amazônica, incluídas hoje em pesquisas sociais e médicas. Objetivo: determinar o grau de compreensão do CI numa comunidade aymara do Peru com um projeto de nutrição escolar. Metodologia: realizou-se um estudo de campo quase experimental, prospectivo, de intervenção educativa em Puno, em 2015. Explicou-se aos líderes aymaras os objetivos e os procedimentos do estudo de nutrição escolar e CI. Fizeram-se um questionário de conhecimentos e práticas de lancheiras saudáveis para crianças aymaras; um programa educativo de nutrição e escolar e dois formulários de CI (simples e com adequação cultural). A documentação foi avaliada por um comitê de ética da pesquisa. Resultados: 70 moradores aymaras participaram do projeto de pesquisa. O grau de compreensão do CI se modificou com significância estatística após a intervenção educativa e a adequação cultural.

Palavras-chave: aymaras; compreensão; consentimento informado; pesquisa; povos indígenas (Fonte: DeCS, Bireme). 


\section{INTRODUCCIÓN}

El progreso de la medicina está relacionado directamente con el desarrollo de investigaciones científicas; sin embargo, la historia de la investigación con seres humanos registra episodios de violación de los principios éticos que deben regirla, como el respeto de la dignidad, la seguridad y el bienestar de los participantes, así como los principios de beneficencia y justicia (1).

En la mayoría de los casos emblemáticos de la investigación con seres humanos controversiales y con faltas de ética, los participantes involucrados fueron personas vulnerables. Según las Pautas Éticas Internacionales para la Investigación Biomédica en Seres Humanos (CIOMS): "Son personas vulnerables las absolutas o relativamente incapaces de proteger sus propios intereses. Específicamente, pueden tener insuficiente poder, inteligencia, educación, recursos, fuerza u otros atributos necesarios para proteger sus intereses” (2).

Dentro del grupo de personas vulnerables se puede incluir a discapacitados mentales, personas en extrema pobreza, prisioneros/refugiados, mujeres embarazadas, niños, minorías étnicas, entre otros (2). Dentro de las minorías étnicas podemos encontrar las llamadas comunidades indígenas o también comunidades nativas o aborígenes (3).

Los pueblos indígenas constituyen entre el $30 \%$ y más del $50 \%$ de la población total de Bolivia, Guatemala, Perú y Ecuador. En cinco países (Perú, México, Guatemala, Bolivia y Ecuador) vive casi el $90 \%$ de los indígenas de la región latinoamericana (4). En Perú, más de ocho millones de personas son indígenas, en su mayoría quechuas y aymara, asentados en la región Andina. En estas comunidades se realizan diversos tipos de investigaciones, y dentro de estas se registran investigaciones sociales, antropológicas, médicas, genéticas, investigaciones con plantas medicinales, etc. (4).

Se han registrado casos emblemáticos de investigaciones con violaciones a los principios éticos en poblaciones originarias o pueblos indígenas. Tal es el caso de la investigación con muestras genéticas en la tribu de Havasupai, una tribu amerindia que habita la región del oeste del Gran Cañón, en el estado estadounidense de Arizona. La investigación analizó temas de predisposición genética a la esquizofrenia que ocasionó graves daños de estigmatización a la tribu indígena. Dicha investigación se desarrolló sin contar con el respectivo consentimiento informado o autorización alguna de la tribu o de sus líderes (3). En algunos casos, los participantes han sido perjudicados o sus derechos han sido vulnerados por los investigadores. Estas situaciones ocurren, muchas veces, porque los participantes no están debidamente informados o no comprenden el objetivo, los riesgos y otros aspectos de la investigación.

Debido a estos antecedentes, es de gran importancia entender que toda investigación con pueblos indígenas o minorías étnicas debe contar con un adecuado proceso de toma de consentimiento informado (CI). Este último, de tipo escrito, es el documento que da fe de la autorización que una persona —o su representante legal- emite para que se le realice un procedimiento médico-quirúrgico o para ingresar a una investigación. El CI es una autorización entendida, competente y voluntaria (5).

En el CI se explican aspectos importantes de la investigación, por ejemplo, el objetivo de la investigación, los 


\section{EN LAS INVESTIGACIONES CON COMUNIDADES INDÍGENAS SE DEBE VERIFICAR LA COMPRENSIÓN DE LOS OBJETIVOS, LOS MÉTODOS DE INVESTIGACIÓN, LOS RIESGOS Y BENEFICIOS, ETC. EXISTE EVIDENCIA DE QUE LOS PARTICIPANTES PROVENIENTES DE COMUNIDADES INDÍGENAS NO ENTIENDEN LA TOTALIDAD DEL Cl.}

riesgos y beneficios posibles, las alternativas adecuadas, el alcance de la confidencialidad, las personas de contacto, la participación voluntaria, los resultados como primera fuente, el derecho de retirarse, entre otros $(3,4)$.

En las investigaciones con comunidades indígenas se debe verificar la comprensión de los objetivos, los métodos de investigación, los riesgos y beneficios, etc. Existe evidencia de que los participantes provenientes de comunidades indígenas no entienden la totalidad del CI. En muchos casos, aceptan participar en una investigación sin haber entendido bien el porqué y el cómo del estudio. Ante esta situación, surge la necesidad de que los investigadores busquen alternativas o mejoras del proceso de toma del CI con la finalidad de que los participantes comprendan toda la información como un acto elemental de respeto, responsabilidad (individual y colectiva), dignidad, autonomía y justicia, que se da en los dos sentidos, tanto para el investigador como sobre el grupo poblacional objeto de la investigación. Estas mejoras pueden comprender la adecuación del CI de acuerdo con el nivel cultural de la población, la traducción del CI a la lengua nativa, el uso de material audiovisual para una mejor comprensión de los individuos, entre otros $(3,5)$.

$\mathrm{Al}$ reconocerse que las comunidades indígenas, a diferencia de otros grupos poblacionales, asumen con total respeto y verticalidad las decisiones tomadas por sus líderes, llamados también caciques mayores y menores, es importante que el equipo de investigación vincule en todo el proceso de investigación a los líderes de la comunidad. Es importante para la investigación, lograr la generación de confianza y establecimiento de relaciones de trabajo y respeto con pobladores reconocidos por ejercer liderazgo formal e informal; además de establecer el intercambio de información, se puede acceder a conocer de cerca la visión del líder sobre necesidades y demandas culturales de la comunidad; además, se pueden lograr consentimientos comunitarios o del líder de la comunidad que, sin embargo, no remplazan el consentimiento individual de cada integrante de estas comunidades indígenas.

Dado lo expuesto, se entiende la necesidad de evaluar si el CI que se aplica en las diferentes investigaciones en poblaciones indígenas peruanas cumple con los requisitos mencionados. De igual manera, es importante averiguar el grado de comprensión en estas comunidades del contenido del CI. Por estas razones, se decidió llevar a cabo este estudio en una comunidad aymara de la sierra peruana.

El objetivo de este trabajo fue determinar el grado de comprensión del CI en la comunidad aymara de Moyapampa, ubicada en el departamento de Puno, Perú, en 
el contexto de una investigación sobre nutrición escolar y loncheras saludables en niños aymaras.

\section{MATERIALES Y MÉTODOS}

\section{Diseño de estudio}

El tipo de estudio es longitudinal, prospectivo, cuasi experimental de intervención educativa. Se realizó entre junio y noviembre del 2015. Se llevó a cabo en la comunidad de Moyapampa, ubicada en el distrito de Juli, provincia de Chucuito, del departamento de Puno. Se encuentra a una altitud de 3884 metros sobre el nivel del mar a 79 kilómetros de la ciudad de Puno, en Perú.

\section{Población de estudio}

En el estudio participó la población aymara de la comunidad de Moyapampa, Puno. Los criterios de inclusión fueron: ser mayor de edad; pobladores de ambos géneros; adultos que pertenezcan a una comunidad aymara; hablar la lengua aymara; permanecer en la evaluación pretaller, sesión de capacitación y evaluación postaller. Los criterios de exclusión fueron: población infantil y personas que no podían participar del estudio en todas sus fases. La muestra fue intencional y abarcó a los líderes de la comunidad y los pobladores aymaras presentes en las reuniones de la comunidad, quienes aceptaron ser parte de la investigación. Se tomó una muestra de 75 individuos de 100 individuos que cumplían los criterios de inclusión. Se excluyeron cinco personas que no desearon completar todas las fases del estudio.

\section{Variables del estudio}

Las variables estudiadas fueron: a) grado de comprensión del CI simple para participar en la investigación sobre nutrición escolar y b) grado de comprensión del CI con adecuación cultural para participar en la investigación sobre nutrición escolar.

\section{Procedimientos}

En la primera parte de la investigación, para evaluar el grado de comprensión del CI, se realizó una preevaluación con un formato simple de CI. En esta se repartió el CI sin explicarles el motivo del documento; solamente se les leyó el CI tanto en español como en aymara. Seguidamente, se les entregó una ficha para evaluar cuánto habían comprendido acerca del Proyecto de Nutrición Infantil y Loncheras Saludables. Las preguntas de la ficha abarcaron temas como título, objetivos, procedimientos, riesgos, beneficios, confidencialidad y participación voluntaria.

En la segunda parte de la investigación se realizó el Taller de Adecuación Cultural, donde se explicaba el significado, la importancia, el contenido y la finalidad del CI. También se les dio a conocer sus derechos como pacientes en un procedimiento médico y como participantes de una investigación médica.

En la tercera y última parte de la investigación, se volvió a leer el CI. Este presentaba la adecuación cultural en ambos idiomas (español y aymara). Finalmente, se les entregó una ficha para evaluar su grado de comprensión del CI.

La ficha de evaluación constó de siete preguntas que versaban acerca de las partes del CI. La calificación se realizó asignando un punto para cada pregunta contestada correctamente y cero puntos para cada pregunta contestada incorrectamente. Si se obtenía al conteo 
final de ninguna a tres preguntas correctas, el grado de comprensión era malo; de cuatro a seis preguntas correctas era regular, y si las siete preguntas eran correctas el grado era bueno.

\section{Aspectos éticos}

El estudio contó con la aprobación de un Comité de Ética de la Universidad Católica de Santa María, Perú. Se obtuvo el permiso de los líderes de la comunidad aymara estudiada. Antes de llevar a cabo la investigación, se tomó el otorgamiento del CI y se aseguró la confidencialidad de los datos personales de los participantes.

Para asegurar el valor social de la investigación, Emanuel, Wendler y Grady, en el artículo “¿Qué hace que la investigación clínica sea ética?” (5), proponen cumplir con el imperativo de desarrollar investigaciones con un resultado valioso para la comunidad. Con este fin se ejecutaron talleres sobre las propiedades nutritivas de los productos que la comunidad aymara cultiva y elabora para su consumo, pues es un tema prioritario de estas comunidades el estado nutricional de sus hijos. En segundo lugar, se dio información acerca de la importancia del consentimiento informado en las investigaciones con sus comunidades, los requisitos de un consentimiento, para que sea libre, informado y voluntario.

\section{Análisis de datos}

Los datos se obtuvieron de la sumatoria de puntos de las fichas para evaluar el grado de comprensión del CI antes del taller y después de este. Los datos obtenidos se codificaron numéricamente para cada participante con un código para antes de la intervención y después de esta.
Todos los datos obtenidos se vaciaron en una base de datos en Microsoft Excel. El análisis estadístico fue procesado en el software SPSS, versión 16, para su posterior análisis y evaluación comparativo entre los resultados de la evaluación pretaller de adecuación cultural (formato de CI simple) y los resultados postaller de adecuación cultural (formato de consentimiento informado con adecuación cultural).

Se evaluó la diferencia entre los puntajes obtenidos antes del taller de adecuación cultural del CI y después de este, con la prueba $\mathrm{T}$ para muestras relacionadas. Se analizó la diferencia mediante la tabla de contingencias con la prueba de McNemar-Bowker.

\section{RESULTADOS}

En la preevaluación (formato de CI simple, sin adecuación cultural), el 74,3\% obtuvo un grado de comprensión malo; el 18,6\%, una comprensión regular, y el 7,1\%, una comprensión buena.

En el análisis del grado de comprensión de los aymaras encuestados acerca del proyecto de investigación, a partir de la información recibida en la preevaluación (formato de CI simple, sin adecuación cultural), se observa mayor frecuencia del nivel malo de comprensión de CI, que corresponde a la moda del grupo. Se observa, asimismo, el mismo nivel malo en la media, que está dentro del intervalo de puntaje (0-3), correspondiente a malo (tabla 1).

En cuanto al análisis del grado de comprensión de los aymaras encuestados acerca del proyecto de investigación, a partir de la información recibida en la evaluación postaller de adecuación cultural (formato de CI con adecuación 
Tabla 1. Frecuencia de calificación de puntajes de la prueba tomada antes del Taller de Adecuación Cultural

\begin{tabular}{|c|c|c|c|c|}
\hline \multicolumn{5}{|c|}{ Preevaluación } \\
\hline $\begin{array}{l}\text { Comprensión. } \\
\text { de CI simple }\end{array}$ & $\mathbf{n}$ & $\%$ & Media & 2,5714 \\
\hline Malo & 52 & 74,3 & \multirow{2}{*}{$\begin{array}{c}\text { Desviación } \\
\text { estándar }\end{array}$} & \multirow{2}{*}{2,2039} \\
\hline Regular & 13 & 18,6 & & \\
\hline Bueno & 5 & 7,1 & Mediana & 2,5 \\
\hline Total & 70 & 100,0 & Moda & Malo \\
\hline
\end{tabular}

Fuente: elaboración propia.

cultural), se observó que los puntajes cambian totalmente, al pasar la moda a la categoría de bueno; entre tanto, la media del grupo se incrementó a 5,87. Además, la mediana indica que la mitad de los puntajes está por encima de 6,5, es decir, la mitad de los participantes obtuvieron un puntaje bueno (tabla 2).

Tabla 2. Frecuencia de calificación de puntajes de la prueba tomada después del Taller de Adecuación Cultural

\begin{tabular}{|c|c|c|c|c|}
\hline \multicolumn{5}{|c|}{ Evaluación Postaller } \\
\hline $\begin{array}{c}\text { Comprensión de } \\
\text { CI con adecuación } \\
\text { cultural }\end{array}$ & $\mathbf{n}$ & $\%$ & Media & 5,8714 \\
\hline Malo & 6 & 8,6 & \multirow{2}{*}{\begin{tabular}{|c} 
Desviación \\
estándar
\end{tabular}} & \multirow{2}{*}{1,4236} \\
\hline Regular & 29 & 41,4 & & \\
\hline Bueno & 35 & 50,0 & Mediana & 6,5 \\
\hline Total & 70 & 100,0 & Moda & Bueno \\
\hline
\end{tabular}

Fuente: elaboración propia.

Respecto a la evaluación de la diferencia del grado de comprensión de los aymaras encuestados acerca del proyecto de investigación, entre los puntajes obtenidos en la preevaluación (formato de CI simple, sin adecuación cultural) y en la evaluación postaller de adecuación cultural (formato de CI con adecuación cultural), según la prueba $\mathrm{T}$ para muestras relacionadas, se observó que existe diferencia estadísticamente significativa. En consecuencia, el Taller de Adecuación Cultural del CI facilitó comprenderlo, pues la diferencia se expresa en el incremento del puntaje de comprensión (tabla 3).

Tabla 3. Determinación de la diferencia entre los puntajes antes y después del Taller de Adecuación Cultural

\begin{tabular}{|c|c|c|c|c|}
\hline \multicolumn{5}{|c|}{ Estadísticos de muestras relacionadas } \\
\hline Taller & Media & $\mathbf{n}$ & $\begin{array}{c}\text { Desviación } \\
\text { estándar }\end{array}$ & $\begin{array}{c}\text { Error típico } \\
\text { de la media }\end{array}$ \\
\hline Antes & 2,5714 & 70 & 2,20389 & 0,26342 \\
\hline Después & 5,8714 & 70 & 1,42362 & 0,17016 \\
\hline
\end{tabular}

Prueba T para muestras relacionadas $=14,150$. $\mathrm{p}=0,0002$.

Fuente: elaboración propia.

Al realizarse la prueba de McNemar-Bowker, para determinar si existió diferencia del grado de comprensión de los aymaras encuestados acerca del proyecto de investigación, entre los puntajes obtenidos en la preevaluación (formato de CI simple, sin adecuación cultural) y en la evaluación postaller de adecuación cultural (formato de CI con adecuación cultural), se observó que una diferencia estadísticamente significativa.

La tabla 4 muestra la reducción de las bajas calificaciones, que fueron remplazadas con altas calificaciones después del taller. 
Tabla 4. Contingencia de las calificaciones antes y después del Taller de Adecuación Cultural

\begin{tabular}{|c|c|c|c|c|c|c|c|c|}
\hline Después & \multicolumn{2}{|c|}{ Malo } & \multicolumn{2}{c|}{ Regular } & \multicolumn{2}{c|}{ Bueno } & \multicolumn{2}{c|}{ Total } \\
\hline Antes & $\mathbf{n}$ & $\mathbf{\%}$ & $\mathbf{n}$ & $\mathbf{\%}$ & $\mathbf{n}$ & $\mathbf{\%}$ & $\mathbf{n}$ & $\mathbf{\%}$ \\
\hline Malo & 6 & 8,6 & 27 & 38,6 & 19 & 27,1 & 52 & 74,3 \\
\hline Regular & 0 & 0,0 & 2 & 2,9 & 11 & 15,7 & 13 & 18,6 \\
\hline Bueno & 0 & 0,0 & 0 & 0,0 & 5 & 7,1 & 5 & 7,1 \\
\hline Total & 6 & 8,6 & 29 & 41,4 & 35 & 50,0 & 70 & 100,0 \\
\hline
\end{tabular}

Prueba de McNemar-Bowker $=57,0$. $\mathrm{p}=0,0004$.

Fuente: elaboración propia.

$\mathrm{Al}$ analizar el grado de comprensión de las diferentes partes del CI, se observa que los riesgos, los beneficios y los objetivos tuvieron la más baja comprensión; mientras que la participación voluntaria, la confidencialidad y los procedimientos obtuvieron un mejor nivel de entendimiento (tabla 5).

Tabla 5. Comprensión de los elementos del consentimiento informado obtenidos en la preevaluación

(formato de Cl simple, sin adecuación cultural)

\begin{tabular}{|c|c|c|c|c|}
\hline \multirow{3}{*}{$\begin{array}{c}\text { Elementos del } \\
\text { consentimiento } \\
\text { informado }\end{array}$} & \multicolumn{4}{|c|}{ Comprensión } \\
\hline & \multicolumn{2}{|c|}{ Sí } & \multicolumn{2}{|c|}{ No } \\
\hline & $\mathbf{n}$ & $\%$ & $\mathbf{n}$ & $\%$ \\
\hline Título & 47 & 67,1 & 23 & 32,9 \\
\hline Objetivo & 20 & 28,6 & 50 & 71,4 \\
\hline Procedimientos & 14 & 20,0 & 56 & 80,0 \\
\hline Riesgos & 12 & 17,1 & 58 & 82,9 \\
\hline Beneficios & 14 & 20,0 & 56 & 80,0 \\
\hline Participación voluntaria & 35 & 50,0 & 35 & 50,0 \\
\hline Confidencialidad & 38 & 54,3 & 32 & 45,7 \\
\hline
\end{tabular}

Fuente: elaboración propia.
Luego de analizar el grado de comprensión de las diferentes partes del CI en la evaluación postaller de adecuación cultural (formato de CI con adecuación cultural) y después de la intervención educativa, se observó que la participación voluntaria, la confidencialidad y los procedimientos obtuvieron un índice mucho más alto de comprensión y entendimiento. Los riesgos, los beneficios y los objetivos obtuvieron una calificación más alta que antes del Taller de Adecuación Cultural, lo que demuestra que la intervención educativa mejoró la comprensión de los elementos del CI (tabla 6).

Tabla 6. Comprensión de los elementos del consentimiento informado después del Taller de Adecuación Cultural

\begin{tabular}{|l|c|c|c|c|}
\hline \multirow{2}{*}{$\begin{array}{c}\text { Elementos del consentimiento } \\
\text { informado }\end{array}$} & \multicolumn{4}{|c|}{ Comprensión } \\
\cline { 2 - 5 } & \multicolumn{2}{|c|}{ Sí } & \multicolumn{2}{c|}{ No } \\
\cline { 2 - 5 } & n & \% & n & \% \\
\hline Título & 59 & 84,3 & 11 & 15,7 \\
\hline Objetivo & 49 & 70,0 & 21 & 30,0 \\
\hline Procedimientos & 57 & 81,4 & 13 & 18,6 \\
\hline Riesgos & 62 & 88,6 & 8 & 11,4 \\
\hline Beneficios & 58 & 82,9 & 12 & 17,1 \\
\hline Participación voluntaria & 63 & 90,0 & 7 & 10,0 \\
\hline Confidencialidad & 63 & 90,0 & 7 & 10,0 \\
\hline
\end{tabular}

Fuente: elaboración propia.

Por último, utilizando el estadístico de McNemar al $95 \%$ de significancia, se observó un incremento del número de personas que comprendieron los elementos del CI antes del Taller de Adecuación Cultural brindado y después de este. El estadístico de McNemar al $95 \%$ de significancia corroboró este dato con un resultado estadísticamente significativo en todos los casos ( $p=0,000)$, lo cual demuestra que sí existe diferencia e incremento 
de la comprensión de cada elemento del CI después del Taller de Adecuación Cultural.

\section{DISCUSIÓN}

En la actualidad, de acuerdo al informe Los pueblos indígenas en América Latina. Avances en el último decenio y retos pendientes para la garantía de sus derechos, de la Comisión Económica para América Latina y el Caribe (Cepal), son 826 pueblos indígenas en América Latina (6). Según países, se distribuyen así: Perú, 85; Brasil, 305; Colombia, 102; México, 78, y Bolivia, 39. La Cepal advierte que muchos de ellos corren el riesgo de desaparecer de manera física; así como sus costumbres, tradiciones y cultura. Es importante señalar que estas cifras no son cerradas: aproximadamente 200 pueblos indígenas originarios optan por el autoaislamiento voluntario en Bolivia, Brasil, Colombia, Ecuador, Paraguay, Perú y Venezuela (6).

En Perú, estos pueblos indígenas están representados, principalmente, por dos grupos étnicos: los aymaras y los quechuas, quienes se distinguen por ser pueblos indígenas agrupados en familias lingüísticas. Los aymaras pertenecen a las regiones de los Andes y del altiplano de América del Sur, principalmente de la región altiplánica peruana (4).

La población indígena en Perú se destaca por ser el grupo más grande de la región, con un total aproximado 6,5 millones de habitantes. Ellos representan el 25\% de población peruana. En cuanto a la distribución de la población indígena en familias lingüísticas, el $83 \%$ corresponde a los quechuas; el 11\%, a los aymaras, y el $6 \%$, a comunidades de lenguas amazónicas. La lengua materna la población indígena aymara es el aymara, aun cuando es el español, en la mayoría de los casos, su segunda lengua e idioma oficial del Perú $(7,31)$.
LUEGO DE ANALIZAR EL GRADO DE COMPRENSIÓN DE LAS DIFERENTES PARTES DEL CI EN la eVALUACiÓN POSTALLER DE ADECUACIÓN CULTURAL (FORMATO DE Cl CON ADECUACIÓN CULTURAL) Y DESPUÉS DE

LA INTERVENCIÓN EDUCATIVA, SE OBSERVÓ

QUE LA PARTICIPACIÓN VOLUNTARIA, LA

CONFIDENCIALIDAD Y LOS PROCEDIMIENTOS

OBTUVIERON UN ÍNDICE MUCHO MÁS ALTO DE COMPRENSIÓN Y ENTENDIMIENTO.

La toma y el otorgamiento del CI es una obligación moral y legal, cuyo cumplimiento es necesario en todos los ámbitos de atención de salud. Su importancia se hace evidente en el contexto de una investigación científica, más aún si se trabaja con poblaciones vulnerables. Esta últimas, por consenso internacional, son aquellas que no pueden otorgar un CI pleno, por las mismas limitaciones asociadas a su condición de vulnerables: bajo nivel educativo, bajo nivel socioeconómico —que dificulta el acceso al servicio de salud-, analfabetismo, poca familiaridad con términos médicos y el propio lenguaje y dialecto $(3,7,8)$. Se debe aclarar que no todos los aymaras tienen las mismas capas de vulnerabilidad; de acuerdo con el contexto, el poblador aymara puede ser más o menos vulnerable (9).

El CI escrito es el documento que busca dar fe de la autorización que una persona o su representante legal emite para que se le realice un procedimiento médico o quirúrgico, o ingrese a una investigación científica (8). Es 
el reflejo del cumplimiento de respeto de la autonomía de los participantes de una investigación (9). En este artículo se sostiene que el CI debería tener la correspondiente adecuación cultural según el paciente, el sujeto de estudio y las comunidades que representan. La adecuación cultural es necesaria para poder cumplir con informar y proteger a los sujetos de estudio plenamente (10).

Los pueblos aymaras, en su mayoría, viven en condiciones de vulnerabilidad. Y dicha característica nos motivó a desarrollar esta investigación, para determinar el grado de comprensión del proceso de la toma y el otorgamiento del CI.

En lo concerniente a los resultados, observamos que en la primera etapa de evaluación de la comprensión del CI (información recibida en la preevaluación: formato de CI simple, sin adecuación cultural) los pobladores aymaras mostraron mayoritariamente inadecuada comprensión del CI, con un total de 91,9\% (malo: $21,6 \%$ y regular: 70,3\%); solamente el 8,1\% alcanzaron el nivel bueno. Estos resultados manifiestan que los pobladores aymaras no conocen y no comprenden con claridad la propuesta y motivo del CI.

Nuestros resultados coinciden con estudios internacionales sobre la comprensión del CI. Investigaciones realizadas con pueblos indígenas africanos, para determinar el grado de comprensión de la información del CI, concluyeron que los valores occidentales no son entendidos en el contexto africano. En África la población, en general — mucho menos las tribus-, no está familiarizada con el concepto de investigación (11). La intervención experimental de nuestro proyecto consistió en la presentación de un video de la toma y el otorgamiento del CI en la práctica médica. Además, se desarrolló un Taller de Adecuación Cultural con diapositivas acerca de la toma y el otorgamiento del CI en la práctica médica y en la investigación. El programa educativo de intervención de adecuación cultural contó con el apoyo de un traductor perteneciente a la comunidad aymara mollapampa. Se resaltaron las diferentes partes y elementos del CI, haciendo hincapié en que el otorgamiento del CI es un proceso que involucra la autorización entendida, competente y voluntaria por parte del participante o su representante legal para ser parte de la investigación.

Un estudio similar fue realizado en Australia; el objetivo era comparar el proceso de comprensión del CI sobre un ensayo clínico de una vacuna neumocócica, conjugada en aborígenes australianas. La muestra estuvo constituida por 20 mujeres aborígenes y 20 mujeres no aborígenes. Se les administró un instrumento de evaluación preintervención, seguido de otra evaluación postintervención. La información otorgada al grupo de mujeres aborígenes fue elaborada y transmitida por investigadores aborígenes, utilizando medios verbales, materiales visuales y escritos. El grupo de mujeres no aborígenes recibió la información estándar del patrocinador. Luego de la intervención experimental del proyecto, fue evidente la mejora en la comprensión del CI, por lo que estos resultados se consideraron satisfactorios (12).

En lo concerniente a la adecuación cultural del CI, la evidencia muestra que existen dificultades en la efectividad de los procedimientos de la toma y el otorgamiento del CI en poblaciones indígenas. Se requieren herramientas adecuadas para mejorar la comprensión del CI, las cuales deben cumplir con el respeto a los principios éticos fundamentales. El CI con adecuación cultural debe tener en cuenta "las consideraciones y limitaciones de los valores culturales locales" (13). 
Las dificultades en la toma del CI en pueblos indígenas no solo se presentan por la gran variabilidad de las lenguas locales y el difícil acceso a traducciones adecuadas, sino también por sus diferentes cosmovisiones de la vida, la salud y la enfermedad; así como por barreras debidas a la falta de familiaridad con temas de salud e investigación. Entre las estrategias para mejorar la comprensión del CI se propone el uso de medios orales, de video y animaciones $(3,13)$. En el presente estudio se utilizaron medios orales, trifoliados con dibujos de personajes y escenarios aymaras; asimismo, se proyectaron varios videos.

Nuestros resultados evidencian que el grado de comprensión de los pobladores aymaras de las diferentes partes del CI no fue uniforme, y de estas los objetivos, los procedimientos y los riesgos fueron los elementos del CI con regular o bajo nivel de entendimiento. Lo anterior coincide con diversas investigaciones llevadas a cabo con poblaciones indígenas y aborígenes, donde se reconoce que el procedimiento de toma del CI y su comprensión son de difícil medición; adicionalmente, es variable cómo se entienden los elementos del CI.

Una revisión sistemática de artículos publicados entre 1961 y 2006 sobre CI en la investigación clínica determinó que solo el $54 \%$ de los participantes en la investigación tenía una buena comprensión del objetivo del estudio; el $50 \%$ entendía la asignación al azar; el $47 \%$, el significado de voluntariedad; el $44 \%$, la posibilidad de retirarse del estudio; el $50 \%$, el significado de los riesgos del estudio, y el $57 \%$, los beneficios del estudio (14).

Nuestro estudio cumplió con las siguientes recomendaciones para llevar a cabo la adecuación cultural del CI: en primer lugar, se entabló una relación de cordialidad entre los investigadores y los líderes de la comunidad aymara mollapampa; se tomaron en cuenta los valores que importaban a la población aymara estudiada; las sugerencias de los representantes aymaras para la redacción del proyecto de investigación y del CI fueron aceptadas. Además, se convocó a un lingüista de la comunidad para la traducción de los documentos. Se indagó sobre las expectativas de la comunidad acerca de la investigación durante el estudio y después de concluido. Para la mejora de la comprensión del CI se desarrollaron trifoliados con dibujos comprensibles con personajes aymaras. Se llevó a cabo un trabajo conjunto entre los investigadores y la comunidad, a fin de respetar su autonomía y entablar relaciones de confianza, mutuo respeto y valorización de la cultura indígena (3,15-18).

La comunidad aceptó como beneficio de la investigación el desarrollo de charlas educativas y la entrega de material educativo de la investigación: Nutrición Adecuada en Niños Aymaras y Preparación de Loncheras Saludables. Los pobladores de la comunidad estuvieron motivados y permanecieron en todo el desarrollo de las charlas, en las cuales se utilizaron varios materiales educativos. La exposición oral fue acompañada de trifoliados con dibujos de escenarios y personajes aymaras, además se proyectaron videos educativos.

Para lograr una adecuada participación de la comunidad es necesario investigar todos los temas relacionados con sus necesidades (19,20). Existe evidencia documentada de investigaciones llevadas a cabo en comunidades indígenas en los cuales no se abordaron los problemas de salud existentes en dichas comunidades $(3,21,22)$. Ese tipo de investigaciones ha dejado en dichas comunidades la sensación de haber sido objeto de explotación. Además, se hizo uso indebido de la información obtenida o de los datos genéticos para llevar a cabo nuevos estudios. 
Consecuentemente, esas investigaciones condujeron a la violación de la confidencialidad, la estigmatización y discriminación de la comunidad indígena participante (23-26). El presente estudio cumplió con los principios éticos de justicia (27) y justicia distributiva, que promueve que los riesgos y beneficios de las investigaciones con seres humanos sean compartidos por todos los grupos de una sociedad (28). Estos principios se plasmaron como beneficio de toda la comunidad con la intervención del programa educativo Nutrición y Loncheras Saludables, al ser un tema de interés en la comunidad. Así mismo, culminado el estudio, en una feria comunal donde participaban pobladores aymaras, se reprodujo la capacitación sobre CI, al tiempo que se velaba por el principio de la solidaridad (29) y respeto $(30,31)$.

Otros aspectos éticos cumplidos en el estudio con la comunidad aymara fueron: protección de la confianza depositada por la comunidad hacia el equipo de investigación y aseguramiento de la confidencialidad de los datos, ya que, en las conversaciones realizadas con los líderes de la comunidad, el equipo de investigación se comprometió a no publicar fotos o utilizar sus datos para otros objetivos. Algunas investigaciones no tomaron en cuenta esta protección ética y quebraron la confianza de la comunidad y de los líderes; así, en el estudio mencionado, llevado a cabo en la comunidad indígena de Havasupai, se les dio la confianza a los investigadores solamente para recoger muestras de sangre para estudiar la "genética de la diabetes", enfermedad prevalente en esta tribu, pero las muestras de sangre, lamentablemente, no se usaron solo para ese propósito, sino para varios estudios adicionales, uno de ellos fue para examinar la genética de la esquizofrenia. Los resultados fueron presentados en varias conferencias y publicaciones. La confianza de la población indígena de la tribu Havasupai en la investigación había sido quebrada para siempre (32).

Finalmente, es necesario recalcar que la comunidad científica y futuros investigadores interioricen la importancia del rol que desempeña la ética de la investigación y los comités de ética en la protección de los sujetos de estudio, además de la participación de los representantes de las comunidades indígenas en la evaluación de los riesgos y beneficios de una investigación que compromete a seres humanos.

El estudio concluye que las comunidades indígenas aymaras comprenden el procedimiento de CI cuando este cuenta con la debida adecuación cultural. Esta requiere entablar relaciones participativas, de confianza mutua, respeto y diálogo con la comunidad indígena.

\section{REFERENCIAS}

1. Aguilera-Guzmán RM, Mondragón Barrios L, Medina-Mora Icaza ME. Consideraciones éticas en intervenciones comunitarias: la pertinencia del consentimiento informado. Salud Ment (Mex) [internet]. 2008;31(2):129-38. Disponible en: http:// www.scielo.org. $\mathrm{mx} / \mathrm{scielo}$.php?script=sci_arttext\&pid=S0185$33252008000200007 \& \operatorname{lng}=\mathrm{es}$

2. Consejo de Organizaciones Internacionales de las Ciencias Médicas (CIOMS), Organización Mundial de la Salud (OMS). Pautas Éticas Internacionales para la Investigación Biomédica en Seres Humanos [internet]. Ginebra; 2002. Disponible en: http://www.bioetica.ops-oms.org/E/docs/CIOMS.pdf

3. Muñoz del Carpio Toia A. Ética de la investigación en poblaciones originarias. En: Cuestiones de bioética en y desde Latinoamérica. Ginebra: Unesco-Thomson Reuters; 2011. p. 411-37.

4. Chuecas A. Lo cultural y lo político en los procesos de globalización: pueblos indígenas. En: Castro C, Cornejo M, editores. 
Acceso a los servicios bibliotecarios y de información en los pueblos indígenas de América Latina [internet]. Lima: Centro Amazónico de Antropología y Aplicación Práctica; 2003. p. 23-30. Disponible en: http://www.iidh.ed.cr/comunidades/ diversidades/docs/div_enlinea/lo\%20cultural.htm

5. Emanuel E, Wendler D, Grady C. What makes clinical research ethical? JAMA. 2000;283:2701-11.

6. Naciones Unidas, Comisión Económica para América Latina y el Caribe (Cepal), Centro Latinoamericano y Caribeño de Demografía (Celade). Los pueblos indígenas en América Latina: avances en el último decenio y retos pendientes para la garantía de sus derechos [internet]. Santiago de Chile: Naciones Unidas 2014. Disponible en: http://repositorio.cepal.org/ bitstream/handle/11362/37222/S1420521_es.pdf?sequence=1

7. Benavides M, Mena M, Ponce C. Estado de la niñez indígena en el Perú. Lima: Instituto Nacional de Estadística e Informática (INEI) y Fondo de las Naciones Unidas para la Infancia; 2010.

8. Muñoz del Carpio A, Sánchez H, López M. Bioética y salud pública en América Latina: vulnerabilidad, pobreza e investigación. En León Correa FJ, Sorokin P, editores. Bioética y salud pública en y para América Latina. Santiago de Chile: Felaibe.

9. Luna F. Vulnerabilidad: la metáfora de las capas. Jurisprudencia Argentina. 2008;4:60-7.

10. Congote A, Rincón HG, Salazar JC. El consentimiento informado de la Fundación Clínica Valle del Lili. Colomb Méd. 1999;30:99-100.

11. Afolabi MO, Okebe JU, McGrath N, Larson HJ, Bojang $\mathrm{K}$, Chandramohan D. Informed consent comprehension in African research settings. Trop Med Int Health. 2014 Jun;19(6):625-42.

12. Russell FM, Carapetis JR, Liddle H, Edwards T, Ruff TA, Devitt J. A pilot study of the quality of informed consent materials for Aboriginal participants in clinical trials. J Med Ethics. 2005;31:490-4.

13. Fajreldin V. Problemas bioéticos de la investigación biomédica con pueblos indígenas de Chile. Acta Bioeth. 2010;16(2):191-7.
14. Falagas ME, Korbila IP, Giannopoulou KP, Kondilis BK, Peppas G. Informed consent: how much and what do patients understand? Am J Surg. 2009;198(3):420-35.

15. Dides Castillo C, Pérez Moscoso MS. Investigaciones en salud sexual y reproductiva de pueblos indígenas en Chile y la ausencia de pertinencia étnica. Acta Bioeth. 2007;13(2):216-22.

16. National Health \& Medical Research Council. Values and ethics: Guidelines for ethical conduct in aboriginal and Torres Strait Islander Health Research [internet]. Australia Government; 2003 [citado 2016 may 13]. Disponible en: https://www. nhmrc.gov.au/guidelines-publications/e52

17. Nishimura A, Carey J, Erwin PJ, Tilburt JC, Murad MH, McCormick JB. Improving understanding in the research informed consent process: A systematic review of 54 interventions tested in randomized control trials. BMC Med Ethics. $2013 ; 14: 28$

18. Kelly J. Is it aboriginal friendly?: Searching for ways of working in research and practice that support aboriginal women. Contemp Nurse. 2006;22(2):317-26.

19. Holmes W, Stewart P, Garrow A, Anderson I, Thorpe L. Researching aboriginal health: Experience from a study of urban young people's health and well-being. Soc Sci Med. Apr 2002;54(8):1267-79.

20. Bailey J, Veitch C, Crossland L, Preston R. Developing research capacity building for Aboriginal \& Torres Strait Islander health workers in health service settings. Rural Remote Health. 2006;6(4):556.

21. Carjuzaa J, Fenimore-Smith JK. The giveaway spirit: Reaching a shared vision of ethical indigenous research relationships. J Educ Controv. 2010;5(2):4.

22. Van Assche K. Gutwirth S. Sterckx S. Protecting dignitary interests of Biobank research participants: lessons from Havasupai Tribe v Arizona Board of Regents. LIT. 2013;5(1):54-84.

23. Drabiak-Syed K. Lessons from Havasupai tribe v. Arizona state university board of regents: recognizing group, cultural, and dignity harms as legitimate risks warranting integration into research practice. J Health Biomedical L. 2010;6:175-225 
24. Hodge F. No meaningful apology for American Indian unethical research abuses. Ethics Behav. 2012;22(6):431-44.

25. Fuentes DD, Revilla LD. Consideraciones éticas para la realización de investigaciones en comunidades nativas de la selva amazónica del Perú. Rev Peru Med Exp Salud Publica. 2007;24(1):51-66.

26. Lott JP. Module three: Vulnerable/special participant populations. Dev World Bioeth. Mar 2005;5(1):30-54.

27. Macklin R. Ética de la investigación internacional: el problema de la justicia hacia los países menos desarrollados. Acta Bioeth. 2004;10(1):27-36.

28. Comisión Nacional para la Protección de los Sujetos Humanos de investigación Biomédica y Comportamental. Informe Belmont: principios y guías éticos para la protección de los sujetos humanos de investigación [internet]. Barcelona; 1979. Disponible en: http://www.bioeticayderecho.ub.edu/archivos/ norm/InformeBelmont.pdf

29. Lolas Stepke F. Vejez y envejecimiento: la solidaridad como principio bioético. Revista Anales. 2012;7(3):129-36.

30. Rogers W, Mackenzie C, Dodds S. Why bioethics needs a concept of vulnerability. Int J Fem App Bioethics. 2012;5(2):11-38.

31. Ribotta B. Diagnóstico sociodemográfico de los pueblos indígenas de Perú [internet]. Comisión Económica para América Latina y el Caribe (Cepal), Fundación Ford; 2010. Disponible en: http://www.cepal.org/celade/noticias/paginas/6/40386/ Peru_julio2010.pdf

32. Dalton R. Havasupai Indian Tribe v. Arizona Board of Regents. Nature; 430:500-2 